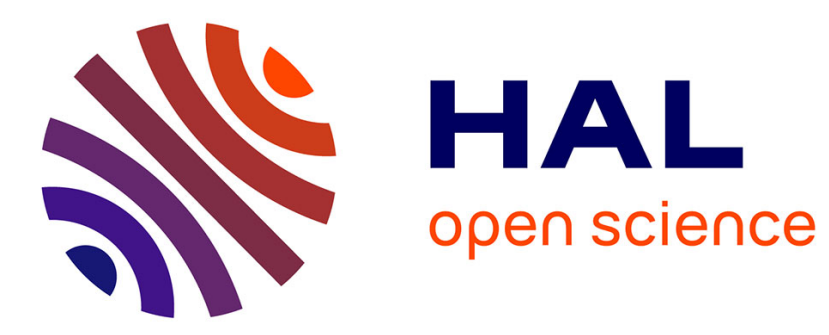

\title{
La détermination de la direction d'arrivée et de la polarisation des atmosphériques siffleurs - Première partie: Les dispositifs expérimentaux
}

Jean Delloue

\section{- To cite this version:}

Jean Delloue. La détermination de la direction d'arrivée et de la polarisation des atmosphériques siffleurs - Première partie: Les dispositifs expérimentaux. J. Phys. Radium, 1960, 21 (6), pp.514-526. 10.1051/jphysrad:01960002106051400 . jpa-00236327

\section{HAL Id: jpa-00236327 https://hal.science/jpa-00236327}

Submitted on 1 Jan 1960

HAL is a multi-disciplinary open access archive for the deposit and dissemination of scientific research documents, whether they are published or not. The documents may come from teaching and research institutions in France or abroad, or from public or private research centers.
L'archive ouverte pluridisciplinaire HAL, est destinée au dépôt et à la diffusion de documents scientifiques de niveau recherche, publiés ou non, émanant des établissements d'enseignement et de recherche français ou étrangers, des laboratoires publics ou privés. 


\title{
LA DÉTERMINATION DE LA DIRECTION D'ARRIVÉE ET DE LA POLARISATION DES ATMOSPHÉRIQUES SIFFLEURS
}

\author{
Première partie : Les dispositifs expérimentaux. \\ Par Jean DELLOUE, \\ Laboratoire de Physique de l'École Normale Supérieure.
}

Résumé. - Les explications avancées pour rendre compte des atmosphériques appelés "siffleurs", se basent sur la théorie magnéto-ionique pour prévoir un mode de propagation particulier des ondes électromagnétiques de fréquence très basse le long des lignes de force du champ magnétique terrestre et à travers les milieux faiblement ionisés de la très haute atmosphère. La portion de l'énergie rayonnée vers le haut par un éclair et survivant aux traversées des couches ionosphériques est canalisée par les lignes de force et ramenée avec une atténuation assez faible à la surface du globe dans l'autre hémisphère. La dispersion importante liée à ce mode de propagation produit un effet de sifflement caractéristique (Storey, 1953).

On a cherché à confirmer cette théorie en déterminant expérimentalement les directions d'arrivée des siffleurs qui doivent faire un angle assez faible avec celle du champ magnétique terrestre, et leur polarisation qui est liée par une formule magnéto-ionique à cet angle.

La direction d'arrivée est déterminée par la mesure des retards entre les signaux engendrés par un siffleur dans deux paires d'aériens identiques placés aux extrémités de deux bases perpendiculaires. Des relais hertziens sur ondes centimétriques sont utilisés pour retransmettre les signaux captés. La polarisation "en projection " est donnée par deux cadres verticaux perpendiculaires et la polarisation dans le plan d'onde s'en déduit si l'on connaît la direction d'arrivée.

Diverses considérations ont conduit à utiliser un appareillage sélectif. Les mesures de retard sont alors ramenées à des mesures de déphasages. La fréquence $5500 \mathrm{c} / \mathrm{s}$ a été choisie parce que les siffleurs y présentent à peu près toujours une amplitude importante tandis que le fond des atmosphériques lointains n'a qu'une amplitude faible.

Les signaux captés par les aériens sont amplifiés par des amplificateurs basse fréquence de gain $80 \mathrm{~dB}$ à faible bruit de fond, identiques et stables, puis après retransmission éventuelle par relais hertziens, par des amplificateurs sélectifs dont les caractéristiques de phase sont semblables et dont la largeur de bande est choisie pour obtenir la meilleure réponse aux variations de fréquence dans le temps le plus fréquemment observées.

Les tensions amplifiées provenant des deux cadres croisés sont appliquées à un oscillographe dit de polarisation. Les tensions engendrées aux deux extrémités de chaque base sont appliquées à un circuit qui affiche instantanément le déphasage sur un oscillographe sous la forme de l'inclinaison d'une droite ou d'une ellipse très aplatie. Une caméra à déroulement continu lent photographie simultanément l'oscillographe de polarisation et les deux oscillographes de déphasages auxquels une modulation de brillance particulière est appliquée.

Les essais sur le terrain ont conduit à modifier à plusieurs reprises l'ensemble de mesures. Des circuits correcteurs de phase ont été introduits et on a dû séparer les parties "directions d'arrivée " et "polarisation " de l'appareillage.

Les résultats obtenus et leur interprétation feront l'objet d'un second article.

Abstract. - The explanations which have been put forward to account for whistling atmospherics are based upon the magneto-ionic theory and suggest a peculiar mode of propagation of very low frequency electromagnetic waves along the lines of force of the earth's magnetic field through the feebly ionized regions of the outer atmosphere. That fraction of the energy radiated upwards by a lightning stroke which survives the crossing of the ionospheric layers, travels along the lines of force and returns to the earth surface in the opposite hemisphere. The high dispersion attached to that mode of propagation accounts for the whistling effect (Storey, 1953).

As a check to the foregoing theory, we tried to determine the directions of arrival of whistlers, which should make a rather small angle with the direction of the magnetic field, and their polarization which is related through a magneto-ionic formula to that angle.

The directions of arrival are determined by measuring the time lag between the signals induced by whistlers in two pairs of identical aerials located at the ends of two perpendicular bases. Microwave radio links are used to re-transmit the induced signals. The "projected "polarization is obtained from two vertical crossed loops, and the plane wave polarization can be deduced provided the direction of arrival is known.

Various considerations led to the choice of a selective apparatus. Time lag measurements are then ordinary phase lag measurements. A frequency of $5500 \mathrm{cps}$ has been selected because the amplitude of whistlers is for most of the time large in that part of the spectrum, while the level of long distance atmospherics is low. 
The signals induced in the aerials are fed to high gain, wide band, low noise amplifiers with stable and identical characteristics, and after being re-transmitted over radio links, to selective amplifiers with identical phase characteristics and a convenient bandwidth to obtain the best response to a signal varying in tone according to the law most often observed. The voltages corresponding to the crossed loops are then applied to a "polarization" oscilloscope. The signal corresponding to the ends of each base are fed to a phase comparator which instantaneously displays the phase lag as the slope of a straight or slightly elliptic pattern on an oscilloscope. A continuous motion slow speed camera photographs simultaneously the polarization oscilloscope and the two phase oscilloscopes to which convenient blanking voltages are applied.

Fields tests of the equipment led to successive alterations of the set-up. Phase correcting networks were introduced and the "polarization" section of the equipment had to be separated from the " directions of arrival " section.

The results obtained and their interpretation will be discussed in another article.

Introduction. - Le travail présenté dans le pages qui suivent est consacré à l'étude de certaines propriétés d'un type de perturbation électromagnétique d'origine naturelle du domaine des très basses fréquences.

Afin de mieux situer le problème, il nous a paru nécessaire de résumer rapidement dans une première partie les traits caractéristiques des diverses perturbations électromagnétiques d'origine naturelle et plus particulièrement de celles affectant la bande des très basses fréquences, puis de préciser l'état actuel des connaissances sur le type de perturbation qui nous intéresse : " l'atmosphérique siffleur ". Nous sommes dès lors en mesure d'exposer l'objet de ce travail : vérifier si les siffleurs se propagent bien le long des lignes de forces du champ magnétique terrestre en déterminant leur direction d'arrivée et si la polarisation observée peut s'expliquer par un tel parcours à travers les couches supérieures de l'atmosphère.

La seconde partie est consacrée à l'exposé des méthodes de mesures utilisées et à la discussion de certains aspects du problème et de leur influence sur la conception de l'appareillage : nécessité de favoriser le plus possible les siffleurs vis-à-vis des autres parasites, problèmes posés par le caractère fugitif des siffleurs et leur occurrence imprévisible, etc...

La troisième et la quatrième partie décrivent les différents éléments de l'appareillage et les modifications successives de l'ensemble de mesures telles qu'elles se sont révélées nécessaires au cours des expériences.

L'interprétation, assez délicate, des diagrammes observés sur les oscillographes de mesure, tant pour la détermination des directions d'arrivée que pour la polarisation, est discutée ensuite. Une méthode simple de représentation des caractéristiques d'un siffleur est proposée.

Enfin, une dernière partie résume les résultats obtenus qui confirment le mode de propagation le long des lignes de force du champ magnétique terrestre et une tentative y est faite de remonter de la polarisation observée aux paramètres affectant la propagation.

\section{Les parasites électromagnétiques d'origine naturelle.}

1-1. - Les perturbations ÉlectromagnéTIQUES BASSE FRÉQUENGE. - Les décharges électriques orageuses sont les sources naturelles les plus importantes de perturbations dans tout le spectre des ondes hertziennes. Elles se manifestent sous la forme de claquements brefs (quelques dizaines de millisecondes) ou de crachements lorsque l'éclair origine a été complexe. Le niveau moyen de ces parasites, assez faible dans la gamme des ondes décimétriques et métriques, s'élève très rapidement lorsqu'on atteint les ondes décamétriques et de radiodiffusion, mais c'est vers les fréquences basses et très basses qu'on observe les niveaux les plus élevés, en particulier aux environs de $10 \mathrm{kc} / \mathrm{s}$. Le phénomène est encore très bref et l'impression auditive est toujours celle d'un claquement mais le spectre présente en général dans cette

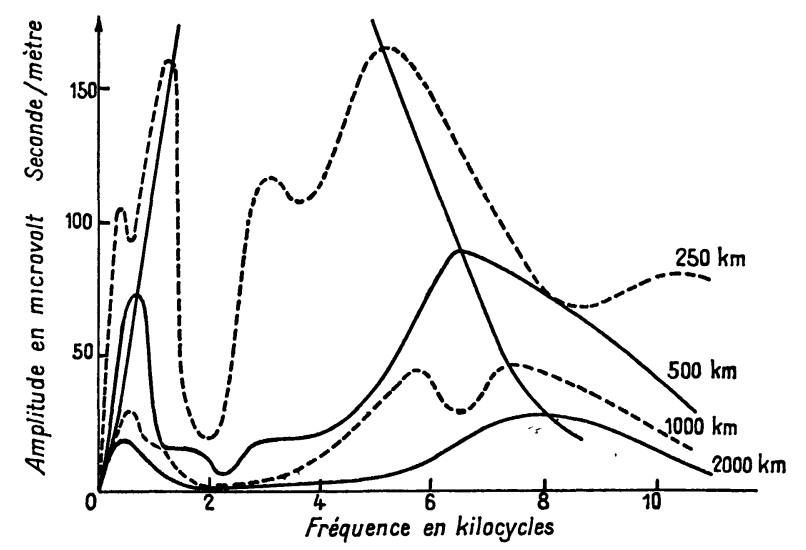

Fig. 1. - Spectre des atmosphériques en fonction de la distance.

gamme de fréquences un aspect remarquable dépendant de la distance du récepteur à l'éclair source (fig. 1) : pour des distances de $2000 \mathrm{~km} \mathrm{ou}$ plus on constate une absence de composantes dans la bande 2000 à $6000 \mathrm{c} / \mathrm{s}$. Nous utiliserons d'ailleurs cette particularité ultérieurement. 
Mais, en plus des atmosphériques classiques apparaissent dans la gamme des très basses fréquences des parasites d'un genre tout à fait différent, de plus longue durée et à tonalité musicale.

Le premier type appelé "tweek» est un atmosphérique musical relativement court dont la durée est de l'ordre de 20 millisecondes. Sa forme d'onde est caractérisée par une oscillation amortie qui suit une impulsion brève. La fréquence de l'oscillation décroît d'abord très rapidement puis reste sensiblement constante à la limite, aux. environs de 1000 à $2000 \mathrm{c} / \mathrm{s}$. Les tweeks sont produits par les arrivées successives des divers échos d'un atmosphérique formés par réflexions successives sur la terre et la basse ionosphère et s'observent principalement au lever et au coucher du soleil.

Le second type appelé " siffleur " consiste sous sa forme la plus courante en un sifflement dont la hauteur décroît depuis des fréquences inaudibles, rapidement tout d'abord, puis plus lentement pour les fréquences basses jusque vers $1000 \mathrm{c} / \mathrm{s}$. Le parasite balaye ainsi plusieurs octaves en l'espace d'une ou deux secondes. C'est à ce genre de parasite que se rapporte notre étude, aussi ferons-nous plus loin une étude approfondie de celles de ses caractéristiques qui sont actuellement bien connues.

Les autres types de parasites basse fréquence sont d'occurrence plus rare et liés à une forte activité magnétique : souffle continu affectant la bande 1000 à $4000 \mathrm{c} / \mathrm{s}$ pendant des heures, " chœur de l'aube » formé de sifflements de hauteurs croissantes sur un fond continu de bruits semblables à des gazouillis, ou siffleurs ascendants à évolution rapide.

1.-2. - Les Parasites siffleurs. - Bien que leur existence soit connue depuis longtemps, leur étude systématique n'a été entreprise que récemment. Cependant, dès 1918 Barkhausen [1] entendit " voler les grenades " dans des appareils de télégraphie par le sol et conclut très vite que la cause de ces parasites était extérieure à l'appareillage. Par la suite, les mêmes phénomènes se manifestèrent dans les lignes téléphoniques à longue distance et dans tout aérien de dimensions assez grandes auquel on connectait un amplificateur basse fréquence. Eckersley [2] s'intéressa à cet aspect de la question et proposa le premier en 1925 une explication basée sur la dispersion d'une impulsion électromagnétique dans une atmosphère d'ions. Barkhausen [3] émit en 1930 une théorie qui, si elle convenait particulièrement au tweek, ne pouvait rendre compte des caractéristiques des siffleurs. C'est néanmoins lui qui, le premier, vit dans la décharge orageuse une origine possible des siffleurs. Vers 1933 Burton et Boardman [4], étudiant des câbles sous-marins, publièrent de nombreux résultats sur les tweeks ainsi que le premier oscillogramme et la première analyse spectrale d'un siffleur. En 1935, Eckersley [5], reprenant son ancienne idée relative à la dispersion d'une impulsion électromagnétique dans l'ionosphère, et supposant que la correction de Lorentz, due à la polarisation du milieu, n'est pas à faire, mit en lumière une possibilité de propagation dans le mode extraordinaire des ondes électromagnétiques de très basses fréquences à travers l'ionosphère et trouva pour la variation de la fréquence d'un siffleur dans le temps une loi en $1 / t^{2}$. Cette loi était confirmée par l'analyse spectrale de Burton et Boardman.

Ce n'est qu'en 1953 que Storey [6] reprend la question du point de vue expérimental et théorique et complète la théorie d'Eckersley par une hypothèse très intéressante. Nous allons examiner ses résultats en détail.

1.-2. 1. - Résultats expérimentaux. - Storey montre qu'on peut classer les siffleurs forts en deux catégories suivant qu'ils sont ou non précédés d'un fort claquement atmosphérique. Au moyen d'un réseau de goniomètres cathodiques, il découvre que ces derniers sont dus à des éclairs et proviennent de zones orageuses distantes de moins de $2000 \mathrm{~km}$ du récepteur.

Analysant au moyen d'un spectrographe basse fréquence les dispersions de nombreux siffleurs enregistrés sur bande magnétique, il vérifie la relation proposée par Eckersley :

$$
t=D f^{-1 / 2}
$$

$D$ étant appelée dispersion du siffleur. De plus, sur un graphique représentant $f^{-1 / 2}$ en fonction de $t$, la droite représentative d'un siffleur coupe toujours l'axe des temps à l'instant où s'est produit le fort claquement cause présumée du siffleur. Enfin, la mesure des dispersions de nombreux siffleurs montre que ceux qui sont précédés d'un fort claquement ont toujours une dispersion approximativement double de celle des siffleurs ne jouissant pas de cette propriété.

Lorsque les siffleurs arrivent en groupe, ce n'est pas nécessairement le seul fait du hasard. Storey distingue les "trains de siffleurs " formés d'un siffleur et de plusieurs échos (jusqu'à une dizaine se succédant à intervalles réguliers avec des amplitudes de plus en plus faibles et des dispersions croissantes. Sur les diagrammes $j^{-1 / 2}(t)$ les droites représentatives concourent en un même point de l'axe des temps et, s'il y a eu un claquement atmosphérique avant le train, il s'est généralement produit à cet instant et les dispersions sont comme 1-2-3-4 tandis que dans le cas contraire elles sont comme 1-3-5-7. Les « siffleurs d'éclair multiple " se suivent irrégulièrement à de courts intervalles, sont d'amplitudes comparables et ont la même dispersion : leur origine est vraisemblablement dans un éclair multiple. Enfin les " paires " ressemblent à un train de deux siffleurs mais 
leurs amplitudes sont du même ordre et leurs dispersions ne sont pas dans un rapport simple.

Une étude des variations systématiques des propriétés des siffleurs par des moyennes portant sur des périodes de l'ordre du mois montre, outre l'irrégularité de leur occurrence, qu'ils se produisent bien plus fréquemment de nuit que de jour et que les siffleurs longs se font entendre surtout l'été et les courts, l'hiver.

1.-2. 2. - La théorie de Storey-Eckersley. - La loi $t=D \cdot f^{-1 / 2}$ résultant de la théorie de Eckersley se trouve vérifiée par l'expérience. Il y a donc tout lieu de penser que les siffleurs sont bien produits par la dispersion d'atmosphériques impulsifs le long d'un trajet ionisé. Mais il reste à déterminer ce trajet, et en particulier à expliquer comment les siffleurs longs, ayant leur source dans une décharge orageuse locale, reviennent dans la région où ils ont pris naissance avec le caractère que nous leur connaissons, et pourquoi ils sont d'autant plus forts que l'éclair-source s'est produit plus près. D'autre part, l'origine des siffleurs courts est à déterminer.

La théorie magnéto-ionique décrit la propagation d'une onde plane monochromatique dans une di. rection donnée, à travers un milieu ionisé et en présence d'un champ magnétique, au moyen de l'indice de réfraction $\mu$ correspondant à une vitesse de propagation $u$. Dans le cas des siffleurs, il s'agit en fait de trains d'ondes qui, lorsque le milieu est dispersif, voyagent avec une vitesse de groupe $v$ qui est différente de $u$. Si le milieu est anisotrope, le train d'ondes se déplace dans une direction fai-

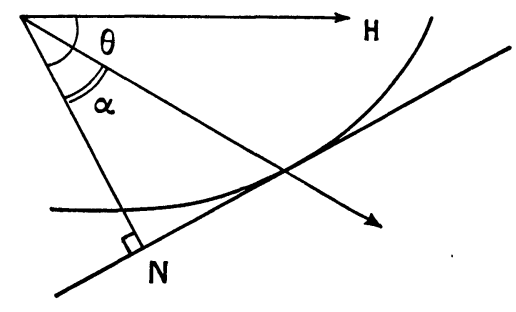

Fig. 2.

sant un angle $\alpha$ non nul avec la normale à la surface d'onde et tel que (fig. 2) :

$$
\operatorname{tg} \alpha=\frac{1}{u} \cdot \frac{\partial u}{\partial \theta}=\frac{1}{2 \mu^{2}} \frac{\partial}{\partial \theta}\left(\mu^{2}\right) .
$$

Dans cette formule $\theta$ est l'angle entre le champ magnétique et la normale et $\alpha$ est positif si la normale est située entre le champ magnétique et la direction de propagation. Dans un milieu à la fois anisotrope et dispersif, le train d'ondes voyage dans la direction de propagation avec la vitesse $V=\varphi / \cos \alpha$.

La formule donnée pour $\mu^{2}$ par la théorie de Appleton-Hartree peut se simplifier dans le cas où les collisions sont négligeables et où l'on admet l'approximation quasi-longitudinale de Booker. Cette dernière est valable aux fréquences basses pour des directions de propagation faisant des angles assez grands avec le champ magnétique et pour des densités électroniques très variables. Il vient alors, en omettant le terme de polarisation de Lorentz et compte tenu des valeurs des paramètres à attendre dans le cas qui nous intéresse :

$$
\begin{aligned}
& \mu^{2}=x / y_{\mathrm{L}} \quad \text { avec } \quad x=p_{0}^{2} / p^{2}=N e^{2} / m f^{2} \\
& \text { et } y_{\mathrm{L}}=\left(p_{\mathrm{H}} / p\right) \cos \theta=(\mathrm{He} / m c) \cos \theta \text {, }
\end{aligned}
$$

$p_{0}$ étant la pulsation critique du milieu et $p_{\mathrm{H}}$ la pulsation correspondant à la gyrofréquence.

L'indice correspondant à la vitesse de groupe est, dans les mêmes conditions :

$$
\mu^{\prime}=(1 / 2)\left(x / y_{\mathrm{L}}\right)^{1 / 2}=(1 / 2) \mu
$$

et l'angle $\alpha$ est défini par :

$$
\operatorname{tg} \alpha=-(1 / 2) \operatorname{tg} \theta .
$$

Le signe moins montre que la direction de propagation est toujours située entre le champ magnétique et la normale à l'onde plane.

$\mathrm{Si}$, au moyen de ces formules, on construit la surface donnant la vitesse de propagation en fonction de la direction de propagation, on voit que, même pour des valeurs de $x / y$ petites, les directions de propagation possibles sont limitées à un cône étroit axé sur le champ magnétique. Le train d'ondes est donc guidé par le champ magnétique dans le milieu ionisé (fig. 3).
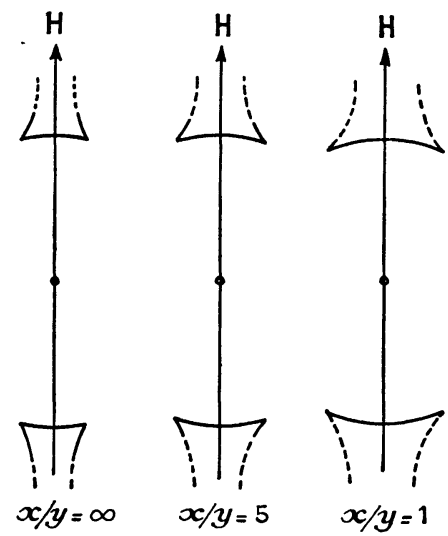

FIG. 3.

Les indices de réfraction pour des fréquences basses sont très grands dans l'ionosphère (de 10 à 300 pour le mode extraordinaire et parallèlement au champ magnétique, plus dès que l'on s'en écarte). Les ondes émises par un éclair et pénétrant dans l'ionosphère suivant une gamme d'angles d'incidence très étendue voient leurs directions de propagation rapprochées énergiquement du champ magnétique par l'effet de réfraction. 
Puis, dans la haute atmosphère, l'effet magnétoionique continue à focaliser l'énergie dans la direction du champ magnétique. On peut par ailleurs montrer que le trajet suivi est pratiquement indépendant de la fréquence.

Storey se basant sur les remarques précédentes suppose que les siffleurs sont des ondes produites par des décharges orageuses qui ont traversé l'ionosphère dans le mode extraordinaire et suivi les lignes de force du champ terrestre. A l'arrivée dans l'autre hémisphère, il reste encore une quantité d'énergie appréciable grâce à la focalisation. La dispersion produit l'effet de sifflement caractéristique.

Un siffleur court capté dans l'hémisphère Nord a pour origine un éclair de l'hémisphère Sud dont l'atmosphérique ordinaire, propagé entre la terre et l'ionosphère, est trop atténué par la longueur du trajet pour être détectable. Un siffleur long capté dans l'hémisphère Nord a pour origine un éclair proche de l'hémisphère Nord (qui produit un atmosphérique audible) les ondes ayant parcouru l'aller-retour le long de la ligne de force avec réflexion sur le sol dans J'hémisphère Sud. La focalisation joue pour rendre le siffleur long audible dans un rayon relativement faible autour de l'éclair source (fig. 4). Les trains de siffleurs s'expliquent

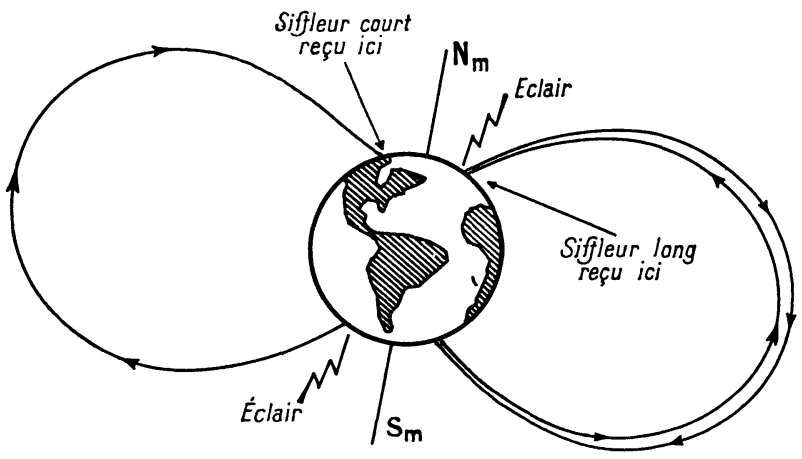

Fig.4. - Trajectoires d'un siffleur long et d'un siffleur court.

par des aller-retour successifs. La dispersion étant proportionnelle au trajet parcouru, les trains consécutifs à un claquement atmosphérique ont des dispersions $2 D, 4 D, 6 D$, etc..., ceux non précédés par un claquement ont des dispersions $D, 3 D$, $5 D$, etc... L'occurrence de siffleurs plus nombreux la nuit que le jour est lié à l'absorption dans la basse ionosphère et l'asymétrie journalière à l'activité orageuse toujours plus forte dans l'après-midi. Enfin, l'abondance de siffleurs longs l'été est liée à la variation saisonnière des orages et celle des siffleurs courts l'hiver à l'alternance des saisons entre les deux hémisphères.
1. 2. 3. - Les conséquences de la thésrie de Storey et l'objet de ce travail. - Une première conséquence importante de cette théorie est de montrer qu'il ne faut pas inclure le terme de Lorentz dans la formule de la polarisation. La validité de ce terme est contestée depuis longtemps et aucune des expériences imagirées pour trancher la question ne s'est révélée concluante.

Une seconde conséquence est l'existence d'un grand nombre d'électrons (400 à 1000 par $\mathrm{cm}^{3}$ ) à des distances de l'ordre du rayon terrestre de la surface du globe. De telles densités sont nécessaires pour expliquer la propagation des siffleurs et les fortes dispersions observées. Ce phénomène semble d'ailleurs avoir été confirmé par des mesures sur la lumiére ${ }^{\bullet}$ zotiacale. Cette ionisation serait due à un apport de matières ionisées en provenance du soleil et expliquerait certaines occurrences simultanées de siffleurs et d'orages magnétiques ou d'aurores.

On imagine aisément l'intérêt présenté par les siffleurs pour l'investigation de la très haute atmosphère jusqu'à plusieurs rayons terrestres. La structure du champ magnétique terrestre, la grandeur et la variation de l'ionisation à ces hauteurs se reflètent dans la dispersion, la forme d'onde et la polarisation des siffleurs. Nous nous sommes donc proposés tout d'abord de vérifier la théorie de Storey-Eckersley en déterminant l'angle d'arrivée des siffleurs par rapport au champ magnétique terrestre et d'entamer l'étude entrevue de la très haute atmosphère terrestre en enregistrant simultanément la polarisation de ces siffleurs afin de tenter de la relier à l'angle d'arrivée. On sait en effet que la théorie magnéto-ionique permet d'évaluer en même temps que l'indice de réfraction $\mu$, Ja quantité $R=-h_{\mathrm{z}} / h_{\mathrm{y}}$ caractérisant le champ électromagnétique de l'onde plane.

\section{Les dispositifs expérimentaux.}

2.-1. - Les méthodes DE MESURE. - La détermination des directions d'arrivée est obtenue par deux mesures de déphasages. Les siffleurs induisent des signaux dans trois aériens disposés aux trois sommets d'un triangle rectangle. La station où s'effectue les mesures est située au sommet de ce triangle et nous l'appellerons station centrale, les deux autres étant les stations auxiliaires. Nous appellerons base l'ensemble constitué par la station centrale et une station auxiliaire. Nous mesurons les déphasages entre Je signal de référence capté à la station centrale et les signaux des stations auxiliaires. Des formules simples relient ces déphasages aux angles (azimuth, élévation) définissant la direction d'arrivée du siffleur. Les fréquences en jeu étant très basses, on doit, pour mesurer un déphasage notable, utiliser des bases relativement longues 
(quelques dizaines de kilomètres). Le problème majeur est alors de retransmettre les signaux captés par les stations auxiliaires à la station centrale sans altérer leurs caractéristiques. Ceci est effectué au moyen de deux ensembles de relais hertziens fonctionnant sur ondes centimétriques.

D'autre part, les aériens de mesures doivent être placés dans des endroits suffisamment dégagés, et éloignés d'accidents du sol trop importants afin de capter une onde aussi peu défor mée que possible. Ces conditions, jointes à celles im posées par l'établissement des liaisons hertziennes rendent très difficile la découverte d'emplacements convenables à des distances relativement grandes.

La mesure de la polarisation se fait à la station centrale au moyen de deux cadres perpendiculaires verticaux. On obtient ainsi en fait la polarisation "en projection », comme nous l'appellerons, mais la connaissance de la direction d'arrivée permet, en principe au moins, de passer de la polarisation en projection à la polarisation dans le plan d'onde.

2.-2. - LE CARACTÈRE SÉLECTIF DES MESURES. - La bande de fréquences où se manifestent les siffleurs est assez grande, de $1500 \mathrm{c} / \mathrm{s}$ à $15000 \mathrm{c} / \mathrm{s}$ et même plus. Il ne semble donc pas a priori s'imposer d'opérer à fréquence fixe. Mais, deux caractères des sjffleurs nous ont amenés à les observer dans une bande relativement étroite de fréquences. Tout d'abord la caractéristique sifflante du siffleur indique que des portions différentes du spectre basse fréquence sont affectées successivement par le siffleur, tandis que les atmosphériques classiques se manifestent simultanément dans toute la bande de fréquences. En opérant en bande large nous capterions donc à un instant déterminé toute l'énergie rayonnée par les atmosphériques à cet instant, mais une portion seulement de l'énergie totale du siffleur. Or, ces atmosphériques perturbent les mesures de phases et de polarisation : nous aurions donc un rapport signal/ bruit (naturel) peu satisfaisant. Cet effet est d'autant plus gênant que les siffleurs sont, la plupart du temps, beaucoup plus faibles que les atmosphériques présents (principalement ceux d'origine proche) : c'est leur variation de tonalité qui permet à l'oreille de les distinguer encore nettement lorsque les appareils de mesure ne donnent que des indications infimes. D'autre part, le désir que l'on a d'éviter toute déformation du front d'onde interdit d'utiliser le relief avoisinant comme protection par effet d'écran.

C'est donc l'idée de protection à l'égard des atmosphériques qui nous a conduits dès le début à opérer dans une bande de fréquences relativement étroite. Or, il est possible de choisir cette bande de façon à minimiser encore l'action des atmosphériques et à renforcer celle des siffleurs.
En $\in$ ffet, la propagation dans le guide d'ondes un peu particulier constitué par la surface de la terre - pratiquement parfaitement réfléchissante - et les couches inférieures de la basse ionosphère - où l'on a plutôt réfraction progressive que réflexion métallique - provoque dans le spectre des atmosphériques ordinaires un affaiblissement d'autant plus marqué des fréquences entre 2000 et $6000 \mathrm{c} / \mathrm{s}$ que la distance de propagation est plus grande. D'autre part, l'observation courante montre que la plus grande partie de l'énergie des siffleurs est localisée dans la partie basse du spectre basse fréquence, de 1500 à $6000 \mathrm{c} / \mathrm{s}$. C'est donc cette région qui est la meilleure. Nous opérons à $5500 \mathrm{c} / \mathrm{s}$ pour des raisons techniques : les relais hertziens employés ayant une bande passante utile allant de 4000 à $28000 \mathrm{c} / \mathrm{s}$, nous avons évité de nous placer trop près de la limite inférieure $4000 \mathrm{c} / \mathrm{s}$ pour éviter les rotations de phase assez importantes dues aux filtres passe-haut utilisés.

L'exploitation de l'appareillage nous a apporté une justification supplémentaire de ce point de vue : le spectre des siffleurs n'est pas constitué par une fréquence pure décroissant dans le temps mais par une bande de fréquences plus ou moins complexe dont la fréquence centrale dérive dans Je temps. En bande large, les motifs observés sur les oscillographes de mesure serajent inexploitables parce que résultant de la superposition de plusieurs motifs élémentaires en général três différents dûs aux diverses composantes spectrales présentes à un instant donné.

2.-3. - LE CHOIX DE LA LARGEUR DE BANDE. Vis-à-vis des circuits sélectifs utilisés, le siffleur se comporte comme un signal de tonalité variant des aiguës aux graves pour lequel on peut définir une fréquence instantanée : c'est la fréquence de la composante de Fourier dont la phase est stationnaire par rapport à la fréquence à l'instant considéré. Dans ces conditions, on peut montrer que la réponse d'un circuit sélectif à une telle tonalité est la meilleure et le rapport signal/bruit est optimum lorsque la largeur de bande $B$ est prise sensiblement égale à la racine carrée de la variation de la fréquence dans le temps. L'emploi d'une largeur de bande plus faible donnerait à la sortie du circuit sélectif un tension dont l'amplitude de crête serait nettement inférieure à celle qu'on obtiendrait pour une fréquence fixe égale à la fréquence propre du circuit sélectif. De plus, dans ce cas, l'enveloppe de la tension de sortie présente des oscillations parasites dont l'amplitude est d'autant plus forte par rapport à la réponse initiale que la largeur de bande est plus faible. Il est préférable d'éviter ces phénomènes.

A la latitude à laquelle nous avons fait nos observations, la dispersion moyenne des siffleurs courts était de l'ordre de $50 \mathrm{~s}^{-1 / 2}$, celle des siffleurs 
longs de $100 \mathrm{~s}^{-\mathbf{1 / 2}}$. Or, si la dispersion est représentée par la loi

$$
\begin{aligned}
& t=D . f^{-1 / 2} \text {, on a } \frac{\mathrm{d} f}{\mathrm{~d} t}=-\frac{2}{\bar{D}} f^{3 / 2} \text { et par suite } \\
& {\left[\frac{\mathrm{d} f}{\mathrm{~d} t}\right]_{5.500 \mathrm{c} / \mathrm{s}}^{\text {court }}=16000 \mathrm{c} / \mathrm{s} / \mathrm{s} \quad \text { et } B \text { opt. }=125 \text { cycles. }} \\
& {\left[\frac{\mathrm{d} f}{\mathrm{~d} t}\right]_{5.500 \mathrm{c} / \mathrm{s}}^{10 n g}=8000 \mathrm{c} / \mathrm{s} / \mathrm{s} \text { et } B \text { opt. }=90 \text { cycles. }}
\end{aligned}
$$

En fait, la largeur de bande utilisée est, pour des raisons techniques, beaucoup plus grande, de l'ordre de 500 cycles. Il est difficile autrement d'obtenir des réponses en amplitude et phase suffisamment semblables pour tous les amplificateurs sélectifs utilisés dans l'appareillage.

2.-4. - RÉalisation DE LA SÉlection DE FRÉQUENCE. - Il faut, en particulier dans les mesures de déphasages, que les caractéristiques de phase et d'amplitude des divers canaux de l'appareillage soient identiques. Cette condition est plus délicate à remplir dans une station mobile et la division de deux des canaux en deux tronçons par les relais hertziens rendrait difficile le contrôle global de la sélectivité de l'appareillage. Nous avons donc préféré travailler en bande large à l'entrée de chaque canal, les parties sélectives de l'équipement étant toutes groupées à la station centrale où il est facile et rapide de vérifier leur bon fonctionnement en cours de mesures. Un avantage de cette méthode est de pouvoir éventuellement changer de fréquence d'étude sans avoir à modifier l'équipement des stations mobiles.

Enfin, l'emploi d'une bande large permet l'écoute et l'identification des siffleurs sans équipement annexe au poste central et aux postes mobiles ainsi qu'une vérification rapide à la sortie des relais hertziens de la bonne marche des canaux auxiliaires.

2.-5. - LE MOdE DE PRÉSENTATION DES RÉSULTATS. - Comme nous l'expliquerons plus bas, les informations fournies par l'appareillage sont disponibles pendant un temps relativement court, de l'ordre au maximum de $1 / 5$ seconde, résultant de la largeur de bande de l'appareillage, et de la " largeur naturelle " des siffleurs. Nous avons donc eu recours à des oscillographes cathodiques pour afficher ces informations •et à un enregistrement photographique pour en permettre l'étude a posteriori.

La présentation de la polarisation en projection s'obtient simplement sur un tube cathodique dit "de polarisation " en formant une figure de Lissajous avec les signaux provenant des deux cadres croisés de la station centrale.

Mais, pour la présentation de chacun des déphasages relatifs aux deux bases, nous avons choisi de traduire directement le déphasage par un angle. Un circuit décrit plus loin fait apparaître sur l'écran du cathodique "de déphasage " un segment ou une ellipse assez fortement aplatie. L'angle de ce segment, ou du grand axe de cette ellipse, avec la direction de déflection correspondant à l'une des paires de plaques du tube fournit la mesure du demidéphasage entre les tensions induites aux deux extrémités de la base correspondante. Cette méthode est infiniment plus commode et plus précise que celle qui consisterait à mesurer le déphasage à partir d'une ellipse de Lissajous ordinaire.

2.-6. - LE PROBLÈME DE L'ENREGISTREMENT DES CARACTÉRISTIQUES DES SIFFLEURS. - L'OCcurrence des siffleurs est totalement imprévisible. D'autre part, la réalisation d'un appareil différenciant les siffleurs des autres types d'atmosphériques en un temps suffisamment court pour que ce renseignement soit utilisable et permette de photographier les caractéristiques de ces siffleurs est très délicate et le fonctionnement peu sûr. Il est donc nécessaire de filmer en permanence les oscillographes : on utilise une caméra $16 \mathrm{~mm}$ modifiée pour permettre un déroulement continu du film à la vitesse de $2 \mathrm{~cm} / \mathrm{s}$.

D'autre part, il faut utiliser une modulation discontinue de la brillance des cathodiques afin de distinguer les motifs successifs traduisant l'évolution du siffleur. Les impulsions de déblocage du wehnelt doivent cependant être assez longues (1/100 sec.) afin que le spot puisse décrire une tranche de siffleur d'une cinquantaine de cycles sur laquelle des atmosphériques d'amplitude équivalente ne produiront que des perturbations partielles ne détruisant pas la cohérence de l'ensemble du motif. La fréquence de récurrence de ces impulsions a été choisie de l'ordre de 30 par seconde pour donner une séparation suffisante entre deux tranches consécutives. Enfin on a trouvé commode de ne pas éteindre complètement le spot entre deux impulsions de brillance : on peut ainsi observer l'enveloppe des siffleurs ce qui facilite leur localisation sur le film.

\section{Les différents éléments de l'ensemble de mesures.}

3.-1. - Relais hertziens. - La partie de l'appareillage qui nous était imposée était les relais hertziens : ce sont des équipements M X 641 C P fabriqués par la C. S. F., et conçus principalement pour l'établissement de tronçons hertziens dans des quartes téléphoniques à courants porteurs. Ils fonctionnent sur ondes centimétriques, entre 1700 et 2000 mégacycles, avec une puissance à l'émission de 300 milliwatts. La porteuse est modulée par des impulsions " en densité " dont la fréquence de récurrence moyenne est 
$115 \mathrm{kc} / \mathrm{s}$. Une voie de service utilise la partie $200-3000 \mathrm{c} / \mathrm{s} \mathrm{du}$ spectre basse fréquence transmis. Le reste de la bande, de $4000 \mathrm{c} / \mathrm{s}$ à $28000 \mathrm{c} / \mathrm{s}$ est réservé aux " courants porteurs ". Le rapport signal/bruit est meilleur que $55 \mathrm{db}$, et des amplificateurs basse fréquence permettent d'utiliser à l'émission des niveaux allant de 2,5 à 0,025 volts.

Ce matériel avait plusieurs caractéristiques intéressantes pour nos mesures. Il est peu encombrant, facile à déplacer et à installer. C'est une considération très importante pour les stations mobiles qui n'existent pas. en permanence, mais doivent être rétablies à chaque séance de mesures. De plus, sa consommation faible le rend particulièrement bien adapté à une alimentation par accumulateurs et commutatrice. La fréquence utilisée et le caractère directif de l'émission évitent toute induction parasite dans les cadres de mesures. Ce phénomène qui s'est manifesté, par exemple, avec des équipements émetteur-récepteurs sur ondes métriques conduit à une saturation dès la première lampe de l'amplificateur basse fréquence avec démodulation et amplification énergique de cette modulation par le reste de l'appareillage. La portée maximum de ces émetteurs est d'environ $60 \mathrm{~km}$ en vue directe, c'est-à-dire de l'ordre de grandeur d'une longueur d'onde pour les fréquences étudiées. Ils sont donc largement suffisants à ce point de vue. Enfin, la voie de service permet la liaison entre les stations mobiles et la station centrale, et l'exécution des opérations de contrôle de l'appareillage en cours de fonctionnement. La bande " courants porteurs " permet la retransmission en "bande large " au-dessus de $4000 \mathrm{c} / \mathrm{s}$ des signaux basse fréquence captés, les parties sélectives de l'appareillage se trouvant toutes à la station centrale.
3.-2. - Aériens. - L'étude de la polarisation en projection nécessite l'emploi de deux cadres croisés. Dans la réalisation initiale chacun de ces aériens fournissait en plus la tension de référence pour la mesure du déphasage de la tension induite dans l'aérien de la station mobile correspondante. Les aériens des stations mobiles étajent donc par nécessité des cadres.

Dans un but de standardisation du matériel, on a donné les mêmes dimensions et les mêmes caractéristiques à tous ces cadres. Les dimensions externes sont faibles afin de permettre le transport de tout le matériel d'une station mobile dans une voiture de tourisme. Les cadres sont carrés, de 1 mètre de côté, 10 centimètres de large, et comportent deux couches de 20 spires chacune de fil $15 / 10$ isolé sous plastique. Les deux couches sont connectées en série, le point milieu étant relié à la masse du cable coaxial bifilaire assurant la liaison avec l'amplificateur : ce montage symétrique permet d'éviter au maximum les effets d'antenne. Sur le terrain, les cadres sont maintenus verticaux par des haubans sur des supports démontables qui les surélèvent d'environ 1 mètre au-dessus du sol.

La hauteur effective de ces cadres à la fréquence $5500 \mathrm{c} / \mathrm{s}$ est d'environ $0,5 \mathrm{~cm}$.

3.-3. - Amplificateurs " Bande large ». Les aériens sont reliés à des amplificateurs basse fréquence qui doivent avoir un gain important, de l'ordre de $80 \mathrm{db}$, une réponse uniforme dans la bande de fréquences étudiée mais aussi une atténuation importante aux fréquences où des émetteurs ondes longues sont susceptibles de se mani fester, et enfin être stable vis-à-vis des variation

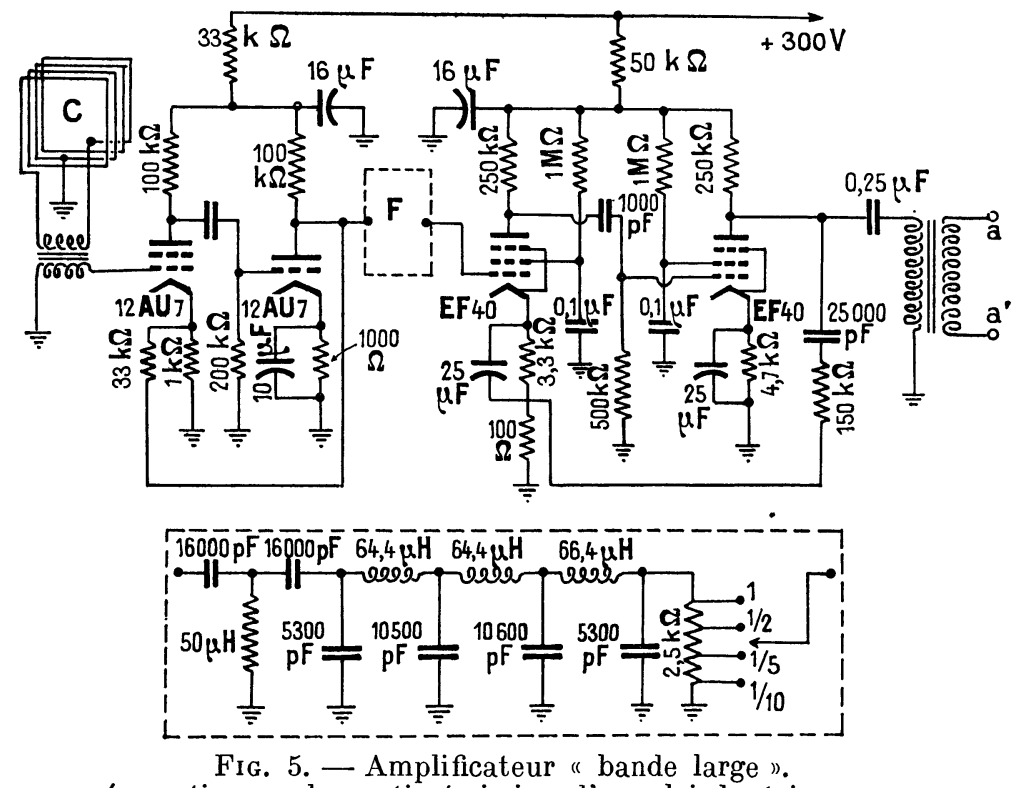

C : cadre ; F : filtre ; a, $\mathrm{a}^{\prime}$ : sortie vers la partie émission d'un relais hertzien ou vers un amplificateur sélectif. 
éventuelles des tensions d'alimentation (surtout pour les stations mobiles).

L'amplificateur utilisé (fig. 5) comporte quatre lampes groupées en deux paires séparées par un filtre. Les deux premières lampes sont des triodes 12 A X 7, afin de minimiser le bruit de fond. La grille de la première triode est attaquée par le secondaire d'un transformateur d'excellente qualité et de grand rapport de transformation (120), dont le primaire, à basse impédance, est relié à l'aérien correspondant. Une contre-réaction assez énergique stabilise l'ensemble des deux triodes.

Le filtre laisse passer la bande de fréquences $2500-11000 \mathrm{c} / \mathrm{s}$. La coupure supérieure évite les interférences des postes ondes longues relativement proches et de fréquences voisines : G. B. R. (Rugby) sur $16000 \mathrm{c} / \mathrm{s}$ et F. U. B. (Houilles) sur $17800 \mathrm{c} / \mathrm{s}$. On a préféré supprimer les fréquences inférieures à $2500 \mathrm{c} / \mathrm{s}$ en raison des nombreuses interférences industrielles rencontrées dans cette gamme.

Les deux dernières lampes sont des pentodes
E F 40 montées avec contre-réaction et attaquées par un diviseur de tension donnant les atténuations 1/1 1/2, 1/5, 1/10. La sortie s'effectue à travers un transformateur d'adaptation d'impédances.

Le gain maximum en tension de l'amplificateur est de 120 000. Le bruit de fond ramené à l'entrée est inférieur à 0,2 microvolts. La saturation dans la position $1 / 1$ est obtenue pour 5 microvolts à l'entrée. Tous les amplificateurs de ce type ont été réalisés aussi semblables que possible, tant vis-à-vis du gain aux diverses fréquences que de la courbe de rotation de phase en fonction de la fréquence. Cette identité est préservée même pour les variations des tensions d'alimentation à attendre dans les stations mobiles.

3.-4. - Amplificateurs sélegtifs. - Un déphaseur résistance-capacité d'impédance moyenne est attaqué par l'entrée basse impédance à travers un inverseur introduisant à volonté un déphasage de $180^{\circ}$ (fig. 6). La sortie du déphaseur se fait à

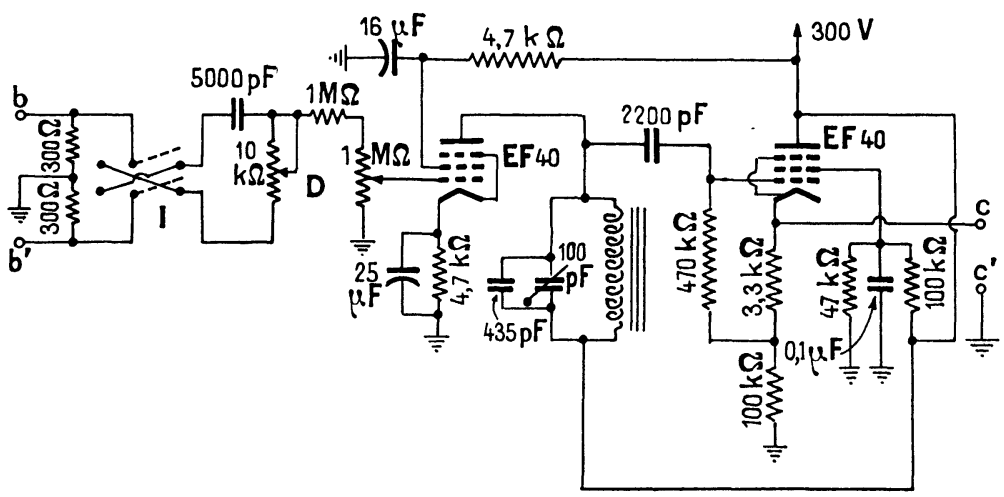

FIg. 6. - Amplificateur sélectif.

$\mathrm{D}$ : déphaseur ; $\mathrm{I}$ : inverseur ; $\mathrm{b}, \mathrm{b}^{\prime}$ : entrée reliée à la partie réception d'un relais hertzien ou à la sortie d'un amplificateur bande large; $c, c^{\prime}$ : sortie vers un calcu- lateur de phase ou vers l'oscillographe de polarisation.

haute impédance, une partie de la charge étant constituée par un potentiomètre de réglage de gain. Ces conditions d'impédance donnent au déphaseur une réponse sensiblement constante en amplitude.

L'étage sélectif est constitué par une pentode E F 40 chargée dans la plaque par un circuit accordé et dont la polarisation est ajustable afin de régler au mieux le point de fonctionnement de la lampe. Les variations minimes des caractéristiques d'un tube à l'autre sont en effet suffisantes pour introduire des anomalies dans la réponse en phase et en amplitude. Il est même nécessaire de sélectionner ces tubes. L'étage de sortie est formé d'une E F 40 montée en cathode follower à très haute i mpédance d'entrée afin d'éviter de charger le circuit sélectif.

Le gain maximum en tension de l'ensemble est de 100. La plage de variation de la fréquence d'accord est de 1000 cycles autour de $5000 \mathrm{c} / \mathrm{s}$. La rotation de phase maximum utilisable donnée par le déphaseur est de $100^{\circ}$. Le réglage de l'ensemble des amplificateurs sélectifs pour l'obtention de courbes de réponse identiques est de loin le plus délicat de l'appareillage. Heureusement, il est relativement stable.

\section{3.-5. - Calculateurs de Phase. - Étant} données les deux tensions

$$
V_{1}=a_{1} \cos \omega t \quad V_{2}=a_{2} \cos (\omega t+\varphi)
$$

on forme les tensions somme et différence et on avance (ou diminue) de $\pi / 2$ la phase de la tension somme par exemple. On effectue ensuite un Lissajous entre la tension ainsi obtenue et la tension différence. Un calcul simple montre qu'on observe 
alors sur l'oscillographe une ellipse dont le grand axe fait un angle $\varphi / 2$ avec une paire de plaques, et dont le rapport des axes est lié homographiquement au rapport des amplitudes des deux tensions initiales :

$$
\frac{B}{A}=\left|\frac{a_{2}-a_{1}}{a_{2}+a_{1}}\right|
$$

Si les deux tensions initiales ont même amplitude $\left(a_{1}=a_{2}\right)$ on observe une droite faisant avec cette paire de plaques un angle $\varphi / 2$. Si les deux tensions sont presque de même amplitude, l'ellipticité observée est un moyen très sensible de vérifier l'égalité des tensions. Enfin, si l'une des tensions est nulle, l'ellipse observée devient un cercle :

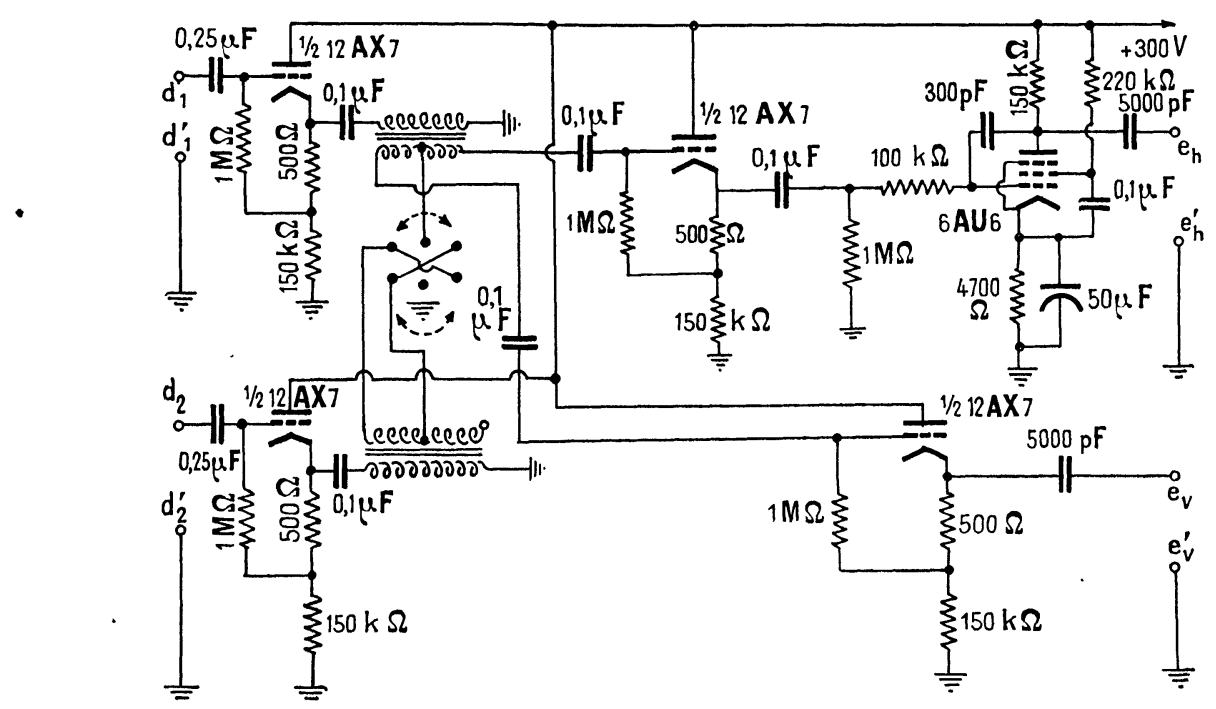

Fig. 7. - Calculateur de phase. $d_{1} d_{1}^{\prime}, d_{2} d_{2}^{\prime}$ : entrées reliées aux amplificateurs sélectifs ; $\mathrm{e}_{\mathrm{h}} \mathrm{e}_{\mathbf{h}}^{\prime}, \mathrm{e}_{\mathbf{v}} \mathrm{e}_{\mathbf{q}}^{\prime}$ : sorties vers les amplificateurs horizontal et vertical de l'oscillographe de phase.

l'aspect de l'oscillographe fournit donc un moyen instantané de contrôle des réglages.

Les tensions somme et différence sont obtenues au moyen d'un montage où les transformateurs sur pots Ferroxcube ont été réalisés avec un très grand soin pour avoir des rapports de transformation égaux ( $f i g .7)$. Ces transformateurs sont attaqués par deux cathodes follower à $12 \mathrm{~A} \mathrm{X} \mathrm{7.} \mathrm{Les}$ tensions somme et différence sont appliquées, après passage à travers deux autres cathodes follower, l'une à une paire de plaques de l'oscillographe de déphasage, l'autre à une 6 A U 6 montée en intégrateur Miller. La tension prélevée dans la plaque est en quadrature avec celle injectée dans la grille, L'intégrateur est réglé à $5500 \mathrm{c} / \mathrm{s}$ mais son fonctionnement est correct dans une bande de 500 cycles. Les erreurs introduites par ce calculateur dans les mesures de phase sont inférieures aux erreurs de lecture sur l'oscillographe.

3.-6. - Les oscillographes de PHASE et DE polarisation. - Les tubes cathodiques utilisés sont du modèle $5 \mathrm{~S}$ P II $\mathrm{A}$ à deux canons et à fluorescence bleue. L'un des tubes est utilisé pour les deux mesures de phase, l'autre, dont un seul canon sert, pour la polarisation. Un petit tube néon placé entre les deux cathodiques permet de localiser les siffleurs sur le film : l'opérateur l'allume quelques instants lorsqu'il perçoit l'un de ces parasites.
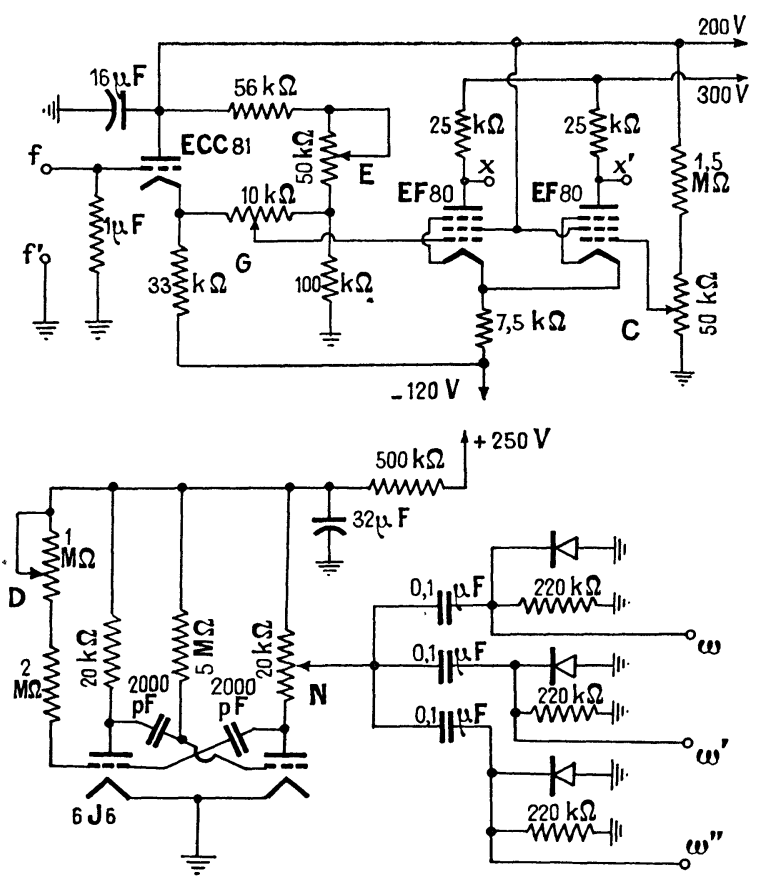

FIg. 8. - Amplificateur d'oscillographe et circuit de modulation de brillance.

$\mathrm{ff}^{\prime}$ : entrée reliée à un amplificateur sélectif ou à un calculateur de phase $; \mathrm{xx}^{\prime}$ : sortie vers les plaques de l'oscillographe ; $\mathrm{C}$ : cadrage ; $\mathrm{G}$ : gain ; $\mathrm{E}$ : équilibrage ; $\mathrm{ww}^{\prime} \mathrm{w}^{\prime \prime}$ vers les wehnelts des oscillographes ; $\mathrm{D}$ :durée, $\mathrm{N}$ : niveau. 
Les sensibilités des deux paires de plaques de ces tubes cathodiques étant voisines, on utilise le même modèle d'amplificateur sur les deux voies (fig. 8) : l'attaque des plaques se fait par deux tubes E F 80 montés en amplificat eur continu différentiel. Un étage préamplificateur continu à E C C 81 comporte les organes de réglage de gain et d'équilibrage. La bande passante s'étend du continu à $150 \mathrm{kc} / \mathrm{s}$ et la rotation de phase avec la fréquence est négligeable jusqu'à $70 \mathrm{kc} / \mathrm{s}$. La sensibilité est de l'ordre de 1 volt crête à crête pour la déflection totale.

Enfin un dispositif de modulation de brillance permet de moduler les whenelts des trois canons avec des ondes carrées de durée et de période réglables fournies par un multivibrateur à $6 \mathrm{~J} 6$.

\section{Les versions successives de l'ensemble de mesures et leur mise en œuvre.}

4.-1. - La première version. - Dans le but de réduire au maximum le nombre d'élémentsnécessaires, on a d'abord adopté un montage où les aériens de polarisation en projection servaient également, en association avecles cadres respectifs des stations mobiles, aux mesures de déphasages. La disposition spatiale des cadres vus à la verticale était donc celle représentée ci-contre ( $f i g .9 a$ ). Le diagramme de l'appareillage était alors assez simple : quatre amplificateurs large bande seulement étaient utilisés. Les portions des quatre canaux comprises

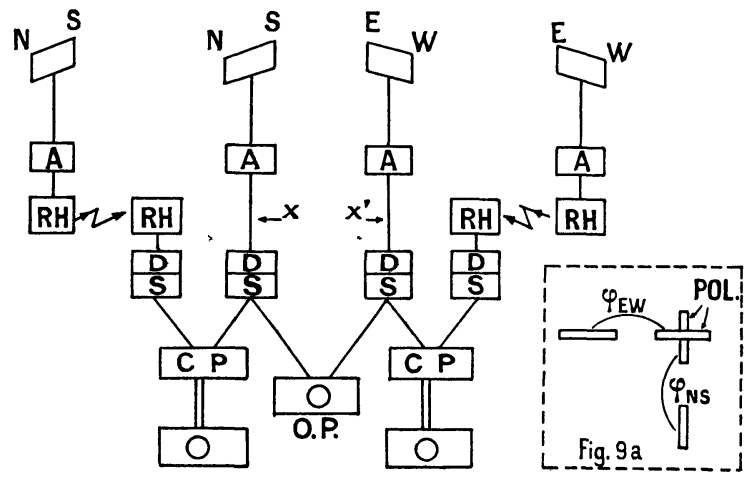

Fig. 9. - Version I.

LÉgende DES Diagrammes DE L'APPareillage. Fig. 9, 11, 12.

A : amplificateur bande large.

$\mathrm{RH} \quad$ : relais hertzien.

C : correcteur de phase.

D : déphaseur.

$\mathrm{S} \quad$ : amplificateur sélectif.

CP : calculateur de phase.

OD : oscillographe de déphasage.

OP : oscillographe de polarisation.

YYY : contacteurs de calibration.

$\mathrm{m}_{1} \mathrm{~m}_{2} \quad$ : commutateurs de mode.

$\mathrm{g}_{1} \mathrm{~g}_{2} \mathrm{~g}_{3}:$ réglages de gain des oscillographes.

$\mathrm{pp}^{\prime}, \mathrm{qq}^{\prime}$ : réglages d'amplitude. entre les aériens et les sorties des amplificateurs sélectifs étaient identiques, à part l'introduction dans deux d'entre eux des deux relais hertziens dont le gain g]obal en tension était choisi égal à l'unité (fig. 9).

Un défaut très gênant qui s'est révélé à l'usage provenait de la rotation de phase introduite par les relais hertziens. La complexité des circuits de ces derniers provoque une variation de déphasage de $100^{\circ}$ par 1000 cycles au voisinage de $5500 \mathrm{c} / \mathrm{s}$. Étant donnée la largeur de bande $(500 \mathrm{c} / \mathrm{s})$ utilisée, on observe une rotation du diagramme de déphasage très importante lorsqu'on applique un signal d'amplitude constante et de fréquence variable à l'entrée des deux canaux correspondants.

D'autre part, le rapport signal sur bruit de l'appareil s'est révélé insuffisant, la majorité des siffleurs observés à l'époque se distinguant à peine $\mathrm{du}$ bruit de fond.

4.-2 - Deuxième version. - La sensibilité de l'équipement a été facilement augmentée en utilisant des transformateurs de couplage aérienscadres de rapport de transformation beaucoup plus élevé. On ne pouvait envisager d'augmenter les dimensions des cadres en raison des servitudes d'espace aux stations mobiles.

Pour compenser la rotation de phase introduite par les relais hertziens, on a inséré aux points $x \mathbf{x}$ du précédent diagramme (fig. 9), deux correcteurs dont la caractéristique de phase était aussi proche que possible de celle des relais hertziens. Les contingences techniques et le désir d'éviter une transformation complète de la réalisation matérielle de l'équipement ont conduit à utiliser des correcteurs purement passifs. Ces circuits, tout en provoquant un déphasage qui varie rapidement avec la fréquence, doivent avoir de plus une réponse en amplitude constante dans toute la bande passante

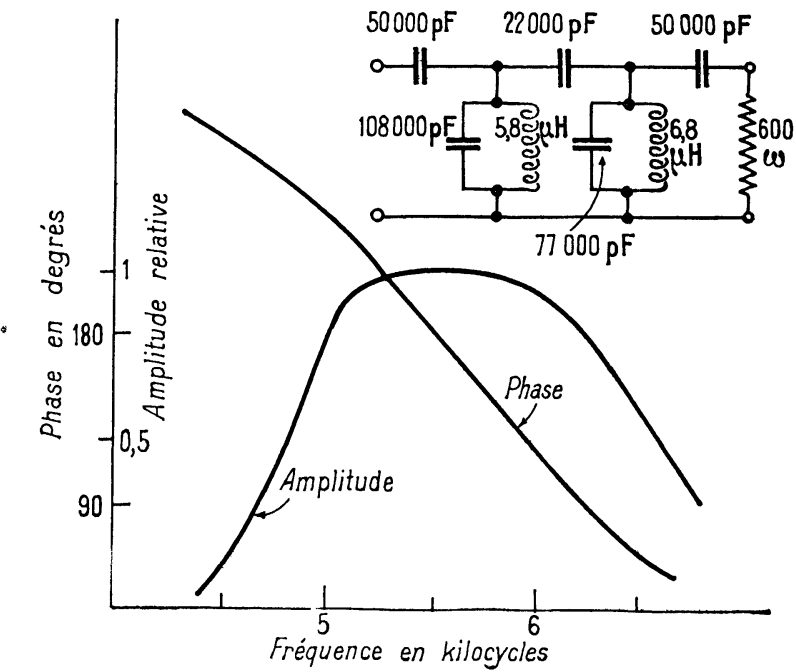

Fig. 10. - Circuit correcteur de phase. 
utilisée : ceci a pu être obtenu en couplant deux cellules en T comme indiqué sur la figure 10. Le problème est rendu très complexe par les variations importantes de l'impédance caractéristique dans la bande passante. Le couplage par condensateur des deux cellules influe aussi beaucoup. D'autre part, de légères retouches sur les divers éléments de chaque cellule permettent de modifier légèrement l'allure de la courbe de phase du correcteur et de la rendre aussi semblable que possible à celle des relais hertziens.

Le contrôle de l'efficacité de ces correcteurs s'effectue en rassemblant tout le matériel en un même point, les stations mobiles auprès de la station centrale, et les relais hertziens fonctionnant sur des distances de quelques mètres. La seule différence avec le fonctionnement réel réside alors dans le déphasage dû au temps de propagation entre l'émetteur et le récepteur de chaque relais hertzien. Les quatre aériens étant débranchés, on injecte à leur place au moyen d'un générateur basse fréquence les quelques microvolts nécessaires et on fait varier la fréquence. On doit alors observer sur les deux écrans de déphasage, deux segments qui varient d'amplitude mais ne tournent pas. Les correcteurs de phase permettent d'obtenir ce résultat à mieux que 10 près dans la gamme $5500 \pm 200 \mathrm{c} / \mathrm{s}$, et à mieux que $2^{\circ}$ près dans la gamme $5500 \pm 500 \mathrm{c} / \mathrm{s}$. Un petit effet résiduel de distorsion $\mathrm{du}$ segment représentatif subsiste néanmoins du côté des fréquences basses, mais pour des amplitudes vraiment très faibles. $\mathrm{Vu}$ l'aspect complexe des oscillogrammes de siffleurs, on peut considérer que le défaut est suffisamment corrigé.

Cependant, deux nouveaux défauts sont apparus à l'usage. Tout d'abord l'augmentation de sensibilité a permis de constater que l'emploi de deux paires d'aériens de directions perpendiculaires pour la mesure des déphasages n'était pas satisfaisant. En effet, si les tensions induites dans les deux cadres croisés de polarisation ne sont pas du même ordre de grandeur, le diagramme de déphasage correspondant à la plus faible des tensions est difficilement lisible. Autrement dit, l'état de polarisation des siffleurs peut rendre l'une ou l'autre des mesures de déphasages impossible. L'observation ayant montré à l'époque que les cadres d'orientation Est-Ouest étaient les plus souvent défavorisés, nous avons décidé de faire les deux mesures de déphasages à partir de trois aériens orientés Nord-Sud. D'autre part, chacun des deux correcteurs étant réglé pour compenser au mieux les défauts du relais hertzien correspondant, les deux canaux de la polarisation en projection n'avaient plus des caractéristiques de phase identiques. Même en se résignant à adopter pour les deux correcteurs une formule moyenne qui compense au mịeux les deux canaux hertziens, il n'était jamais possible de les rendre suffisamment identiques pour que les mesures de polarisation n'en souffrent pas.

4.-3. - Troisième version. - La nouvelle disposition spatiale des cadres devint alors celle représentée sur la figure $11 a$. Le diagramme d'appareillage en était sensiblement modifié et perdait son aspect symétrique. Un amplificateur sélectif supplémentaire était relié à l'amplificateur large bande Nord-Sud de la station centrale par l'intermédiaire d'un seul correcteur de phase et constituait ainsi le canal de référence pour les deux mesures de déphasage (fig. 11).

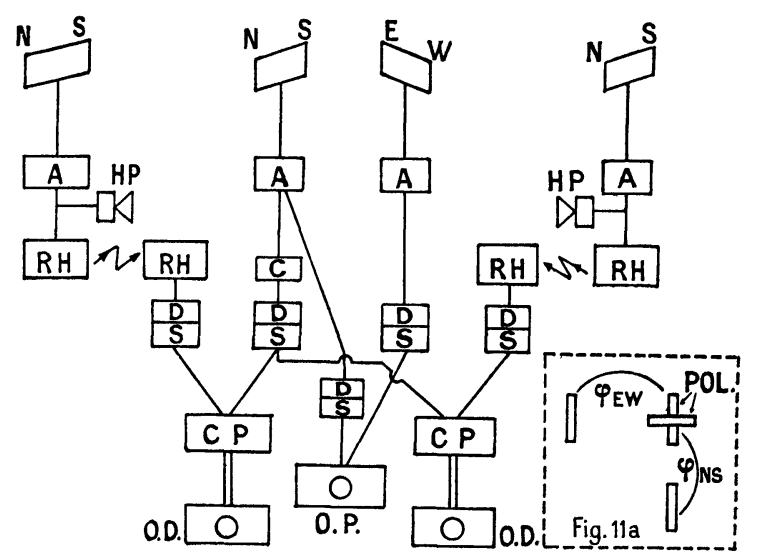

Fig. 11. - Version III.

Mais cette nouvelle version s'est révélée rapidement peu satisfaisante car les contigences de réalisation ne permettaient pas de coupler l'amplificateur large bande Nord-Sud au correcteur de phase par une lampe d'isolement. Ce dernier ayant une impédance d'entrée extrêmement. variable en

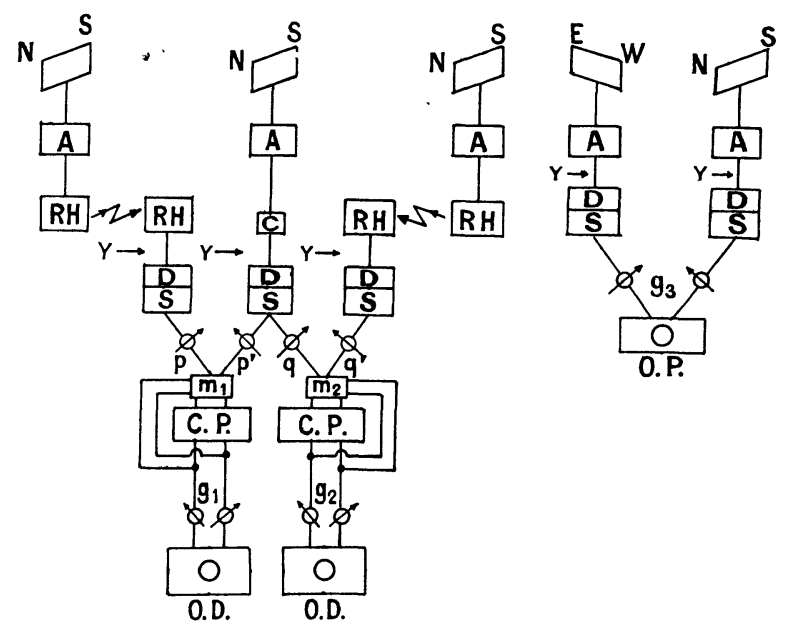

Fig. 12. - Version IV.

A gauche partie "déphasages ", à droite partie " polarisation ". 
fonction de la fréquence, réagissait sur le canal de polarisation sur lequel il était branché et rendait les mesures de polarisation inutilisables.

4.-4. - Quatrième version. - Nous avons alors résolu de séparer complètement la partie " polarisation " de la partie " déphasages " aboutissant ainsi au diagramme de la figure 12. Nous y voyons un cadre, deux amplificateurs large bande et un amplificateur sélectif de plus que dans la première version. Mais cette complication du matériel n'affecte pas les stations mobiles et l'indépendance des mesures, et donc celle des réglages, est assurée. Cet ensemble fonctionne parfaitement.

4.-5. - Les OPÉRATIONS DE CONTROLE. 4.-5. 1. - Contrôle partiel (réalisable même en cours de mesures). - Il est possible de contrôler rapidement le fonctionnement de la partie de l'appareillage située au delà des entrées des amplificateurs sélectifs : des contacteurs indépendants, dits de calibration, permettent d'isoler complètement chaque voie, ou bien de la relier à un générateur basse fréquence. D'autre part, deux commutateurs dits de mode $m_{1}$ et $m_{2}$, permettent d'observer sur les oscillographes de phase, soit l'une ou l'autre des tensions de sortie des amplificateurs sélectifs, soit un diagramme de Lissajous entre ces deux tensions, soit le fonctionnement normal à travers ie calculateur de phase. Enfin les oscillographes sont munis de réglages de gain $g_{1} g_{2} g_{3}$.

On effectue sur la partie " déphasages " les opérations suivantes :

a) On règle successivement les amplificateurs sélectifs en mettant les contacteurs de calibration sur la position "générateur basse fréquence " et en observant les tensions de sortie au moyen des contacteurs de mode, puis on les retouche en les observant deux par deux en Lissajous pour obtenir un segment d'amplitude variable mais de direction fixe quand la fréquence varie.

b) On met le contacteur de calibration de la voie commune sur la position "générateur basse fréquence ", les deux autres commutateurs étant sur ( isolé ") et les commutateurs de mode sur "calculateur ". On doit alors observer sur chaque oscillographe un cercle et, au besoin, on amène l'ellipse observée à devenir un cercle en agissant sur les réglages de gain de l'oscillographe correspondant $\left(g_{1}\right.$ ou $\left.g_{2}\right)$.

c) On met les trois contacteurs de calibration sur la position "générateur basse fréquence " et les deux contacteurs de mode sur "Lissajous ": on observe deux ellipses qu'on transforme en droites au moyen des déphaseurs à l'entrée des amplificateurs sélectifs. On amène ensuite ces droites à faire un angle de $45^{\circ}$ environ avec les directions des paires de plaques des oscillographes au moyen des potentiomètres de réglage d'am. plitude $p p^{\prime}, q q^{\prime}$.

d) On met enfin les commutateurs de mode sur la position "calculateur ": il apparait une droite ou une ellipse très allongée qu'on transforme en droite en retouchant légèrement $p$ ou $q$. Ces droites peuvent être horizontales ou verticales suivant la position des inverseurs de déphaseurs. On choisit l'orientation la meilleure pour la lisibilité des diagrammes de siffleurs comme expliqué plus loin.

Par construction, si l'on agit sur les déphaseurs, toute augmentation du retard de phase d'un canal à relais hertzien par rapport au canal de référence de la station centrale fait tourner la droite correspondante dans le sens des aiguilles d'une montre.

Le réglage de la partie "polarisation » est beaucoup plus simple : seule l'opération $a$ ) est à effectuer. On règle ensuite les gains de l'oscillographe de polarisation pour que le segment observé fasse un angle de $45^{\circ}$ avec les directions des paires de plaques de l'oscillographe de polarisation.

4.-5. 2. - Contrôle total. - Cette opération nécessite, comme expliqué au paragraphe "deuxième version " de faire fonctionner tout l'appareillage au même endroit. Elle permet de contrôler la marche correcte des tronçons de l'équipement inaccessibles en cours de mesures. En fait, le seul réglage est celui de la tension de sortie des relais hertziens, ceux-ci ayant un gain différent de l'unité. On effectue l'opération $d$ ) précédente, en transformant l'ellipse allongée par retouche du potentiomètre de gain. Si l'on fait varier la fréquence du générateur basse fréquence, on observe, en général, en plus de la variation d'amplitude, une très légère distorsion du segment, tandis que sa direction ne change pas. On retouche, si nécessaire, les déphaseurs pour obtenir des segments de déphasage nul exactement verticaux ou horizontaux. Ce contrôle total est une opération très longue exigeant la mise en batteries de tout l'appareillage. Elle n'est pas effectuée fréquemment et l'expérience montre qu'elle est heureusement rarement utile car la stabilité des réglages est très grande.

Manuscrit reçu le 20 novembre 1959. 